\title{
Molecular Analysis of Metabolic Pathway with Graph Transformation
}

\author{
Karsten Ehrig^, Reiko Heckel, and Georgios Lajios ${ }^{\star \star}$ \\ Department of Computer Science, University of Leicester, United Kingdom, \\ \{karsten, reiko,gl51\}@mcs.le.ac.uk
}

\begin{abstract}
Metabolic pathway analysis is one of the tools used in biology and medicine in order to understand reaction cycles in living cells. A shortcoming of the approach, however, is that reactions are analysed only at a level corresponding to what is known as the 'collective token view' in Petri nets, i.e., summarising the number of atoms of certain types in a compound, but not keeping track of their identity.

In this paper we propose a refinement of pathway analysis based on hypergraph grammars, modelling reactions at a molecular level. We consider as an example the citric acid cycle, a classical, but non-trivial reaction for energy utilisation in living cells. Our approach allows the molecular analysis of the cycle, tracing the flow of individual carbon atoms based on a simulation using the graph transformation tool AGG.
\end{abstract}

\section{Introduction}

From the beginning biology has been one of the main application areas of graph transformations [3]. In recent years this line of research has been renewed by proposals for modelling recombination of DNA sequences in cells [7] and other biochemical reactions $[10,12]$.

One major argument in favour of graph transformation-based models for biological systems and chemical reactions is their inherent concurrency, allowing reactions to take place simultaneously as long as they involve different resources and to keep track of causal dependencies and conflicts between them. So far, little of the concurrency concepts available for graph transformation systems have actually been applied in this area. This paper can be seen as a first attempt to identify interesting questions and possible solutions based on a well-known, but non-trivial case study.

In particular we are interested in the analysis of causal dependencies between biochemical reactions. Given a metabolic pathway (a sequence of reactions) we would like to be able to trace the history of particular atoms or molecules. This is relevant, for example, when trying to anticipate the outcome of experiments

* Work was partially supported through the IST-2005-16004 Integrated Project SENSORIA: Software Engineering for Service-Oriented Overlay Computers.

** Research was partially funded by European Community's Human Potential Programme under contract HPRN-CT-2002-00275, [SegraVis]. 
using radioactive isotopes of such atoms. Such questions have been crucial to the detailed understanding of the nature of reactions like the citric acid cycle.

To be able to answer them we propose a new hypergraph model for chemical compounds which refines the classical representation in terms of structural formulae in two different ways.

- Our representation keeps track of the identity of atoms or molecular components by means of the identities of hyperedges. In contrast, when writing down chemical reactions with structural formulae, the identities of the reacting atoms are not explicitly represented in the notation. In situations where several atoms of the same element are involved, this lack of information leads to ambiguity as to where a new atom is placed in the resulting molecule. Our graph transformation-based model allows to track atom identities by graph homomorphisms between the graphs representing the compounds before and after the reaction.

This refinement is comparable to the relation of graph transformation systems and place-transition Petri nets in the collective token view. While the latter record and change the numbers of tokens on certain places (resulting in the rewriting of a multiset of places), graph transformation allows the rewriting of sets of nodes (and edges), each with their individual identity.

- Modelling atoms as hyperedges, each connected to an ordered sequence of nodes, the relative spatial orientation of different molecular components is recorded through the ordering of the nodes connected to a hyperedge.

Using this model we are able to trace the dependencies between different steps in the reaction based on individual atoms and their spatial arrangement. The approach is illustrated by simulating the (at that time surprising) outcome of a classical experiment that led to a deeper understanding of the citric acid cycle. We also provide an encoding of the model in terms of attributed bipartite graphs that can be implemented in the AGG system for simulation and analysis.

The paper is organised as follows. In the next section, we introduce our running example, the citric acid cycle, and explain why the exact configuration of atoms in 3-dimensional space is important for understanding biochemical reactions properly. Our formal model, based on hypergraph transformation systems, is presented in Section 3. In Section 4, we show that the carbon flow in the citric acid cycle can be analysed with the graph transformation tool AGG. A conclusion closes this paper.

\section{Molecular Analysis}

\subsection{Basic Reactions}

Metabolic pathway analysis is one of the tools in biology and medicine in order to understand reaction cycles in living cells. A shortcoming of the approach, however, is that reactions are analysed at the level of structural formulae, summarising the number of atoms of certain types in a compound without keeping 
track of their identity. Molecular analysis aims at understanding chemical reactions at the level of individual atoms or component molecules.

As an example consider the citric acid cycle (also known as the tricarboxylic acid cycle, the TCA cycle, or the Krebs cycle) [13]. This cycle is a series of chemical reactions of central importance in all living cells that utilise oxygen as part of cellular respiration. Starting with acetyl-CoA, one of the resulting products of the chemical conversion of carbohydrates, fats and proteins, the citric acid cycle produces fast usable energy in the form of $N A D H, G T P$, and $\mathrm{FADH}_{2}$ which are precursors of the well known adenosine-tri-phosphate (ATP). The citric acid cycle is shown in Fig. 1.

Fig. 2 shows reaction 1 of the citric acid cycle in detail, focussing on the molecular interactions between oxaloacetate and acetyl-CoA. Acetyl-CoA derived from pyruvate is the output of the metabolism of glucose known as glycolysis and therefore of major importance for the energy metabolism in living cells. CoA known as coenzyme A is just a transport agent for the acetyl group as input for the critic acid cycle.

But how is the metabolism of the acetyl group in the citric acid cycle? The pathway analysis in Fig. 1 shows the acetyl group as input of reaction 1 (see Fig. 2). To analyse the cycle more precisely the first important question is: How is the acetyl group metabolised in the cycle, i.e. in which output agents can we find the two C-atoms of the input acetyl group? Taking a first look would provide the answer: The acetyl group is metabolised into two $\mathrm{CO}_{2}$ molecules. But our further analysis will show that after one cycle the C-atoms of the acetyl group are still in the agents of the cycle.

To analyse this problem more precisely, we have to go one step further in the molecular constitution of the agents. Before we introduce the spatial constitutions of molecules in general in Section 2.2, we would like to introduce this issue with respect to our example.

The output agent of reaction 1, citrate, has two $\mathrm{CH}_{2} \mathrm{COO}^{-}$groups, one on the top and one on the bottom (see Fig. 2). To fit into the enzyme aconitase catalysing reaction 2 (see Fig. 3), only the $\mathrm{CH}_{2} \mathrm{COO}^{-}$group marked with 3 is able to fit into the enzyme due to 3 -dimensional spatial relations (see Fig. 4). This utilised $\mathrm{CH}_{2} \mathrm{COO}^{-}$group will provide the resulting agent $\mathrm{CO}_{2}$ in reaction 4. But the $\mathrm{CH}_{2} \mathrm{COO}^{-}$group provided from the Acetyl-CoA in the beginning of the cycle is not utilised in this reaction. So the main remaining question for our molecular analysis is: Where is the acetyl group metabolised in the citric acid cycle?

This question is important for a deeper analysis of the citric acid cycle and can not be directly answered with the current pathway analysis techniques. Biological pathways are like a black box: We can measure the input and output agents but we know nothing about the reactions in between. To analyse them we have to go down to the molecular level to observe how input agents are utilised during the reaction process. As we have seen in reaction 2, reactions in living cells are more complicated than classical chemical reactions because most of the biological reactions are catalysed by enzymes accepting only special molecular structures. 


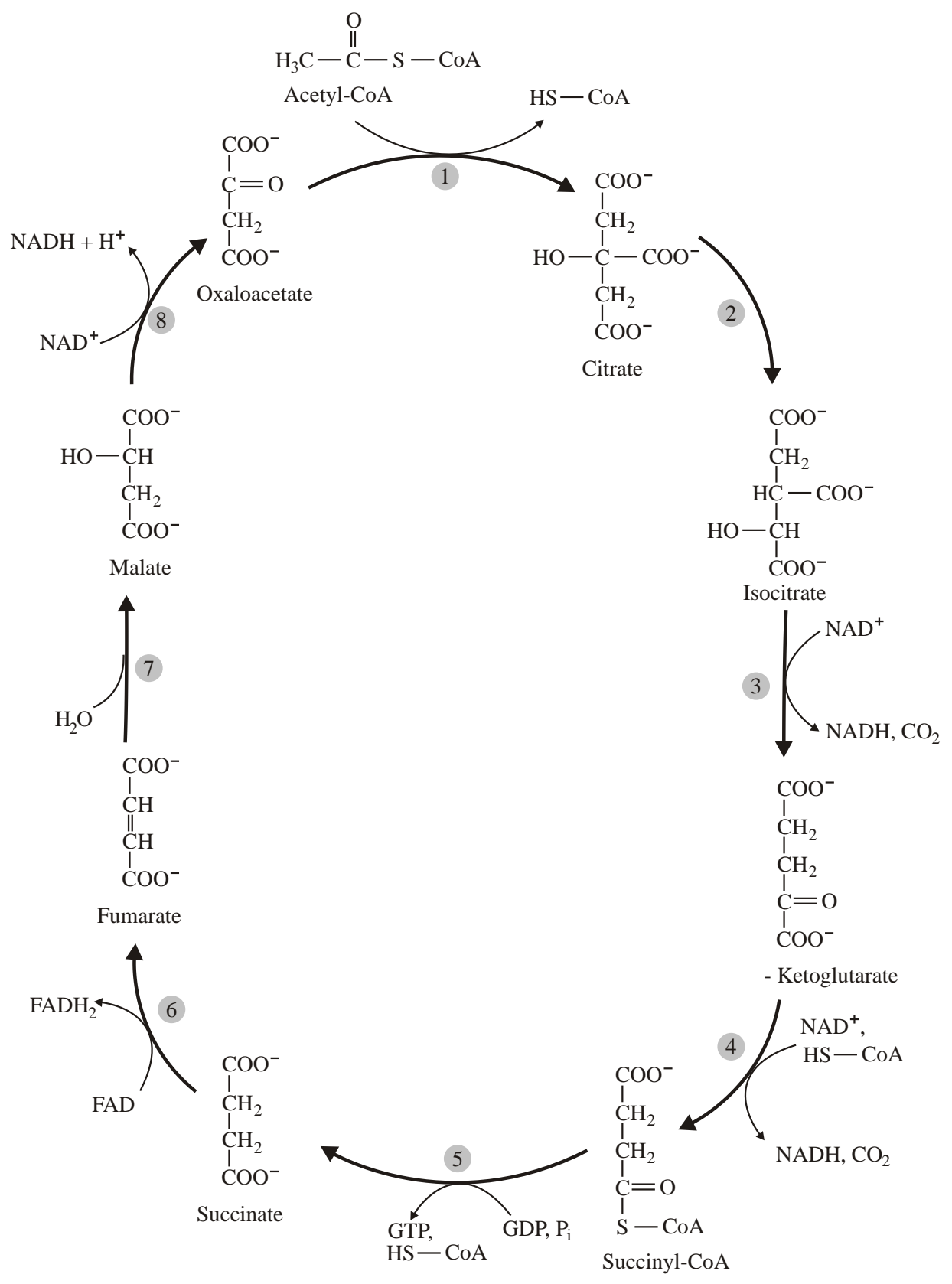

Fig. 1. Citric Acid Cycle 
Apart from the structural formula also the 3-dimensional configuration of the molecules has to be taken into account. To model the configuration we need suitable techniques that can be provided by graph transformation as shown in Section 3.

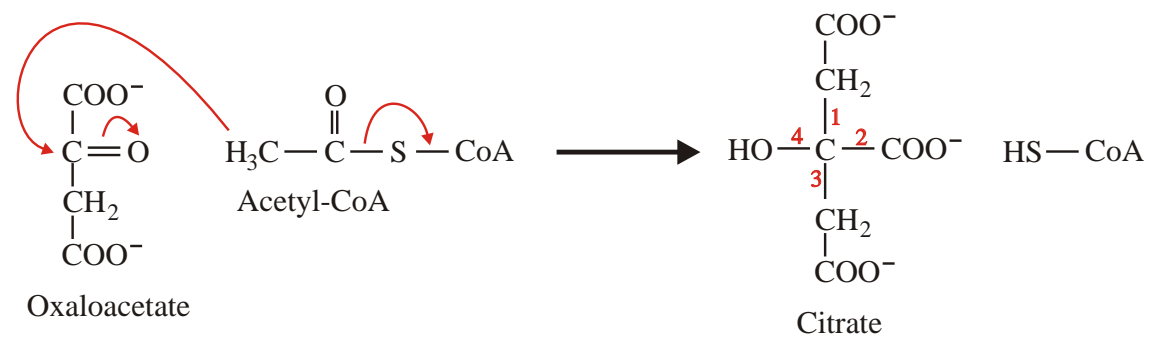

Fig. 2. Reaction 1 of the citric acid cycle

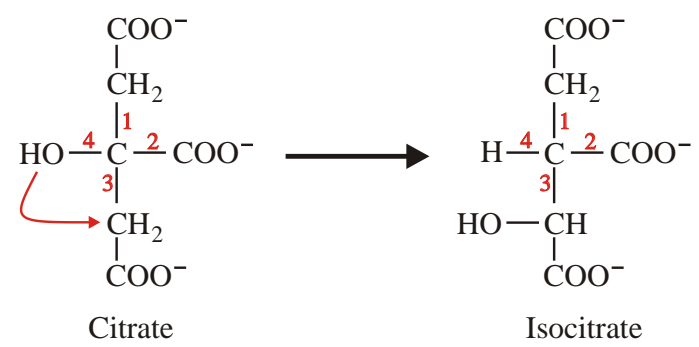

Fig. 3. Reaction 2 of the citric acid cycle

\subsection{Spatial Configuration}

Spatial configuration plays a key role in many chemical reactions. The arrangement of atoms of a molecular entity in space distinguishes enantiomers which have different chemical properties. They often smell and taste differently, and the difference with respect to their pharmacological effect can be serious, as receptors in the human body interact only with drug molecules having the proper absolute configuration. Chiral molecules are mirror images of each other, but can not be superimposed by translation and rotation. Figure 5 shows a sample chiral molecule: glyceraldehyde. The entantiomers L-glyceraldehyde and D-glyceraldehyde act like left and right hands, which are equal except for their arrangement in 3-dimensional space. 


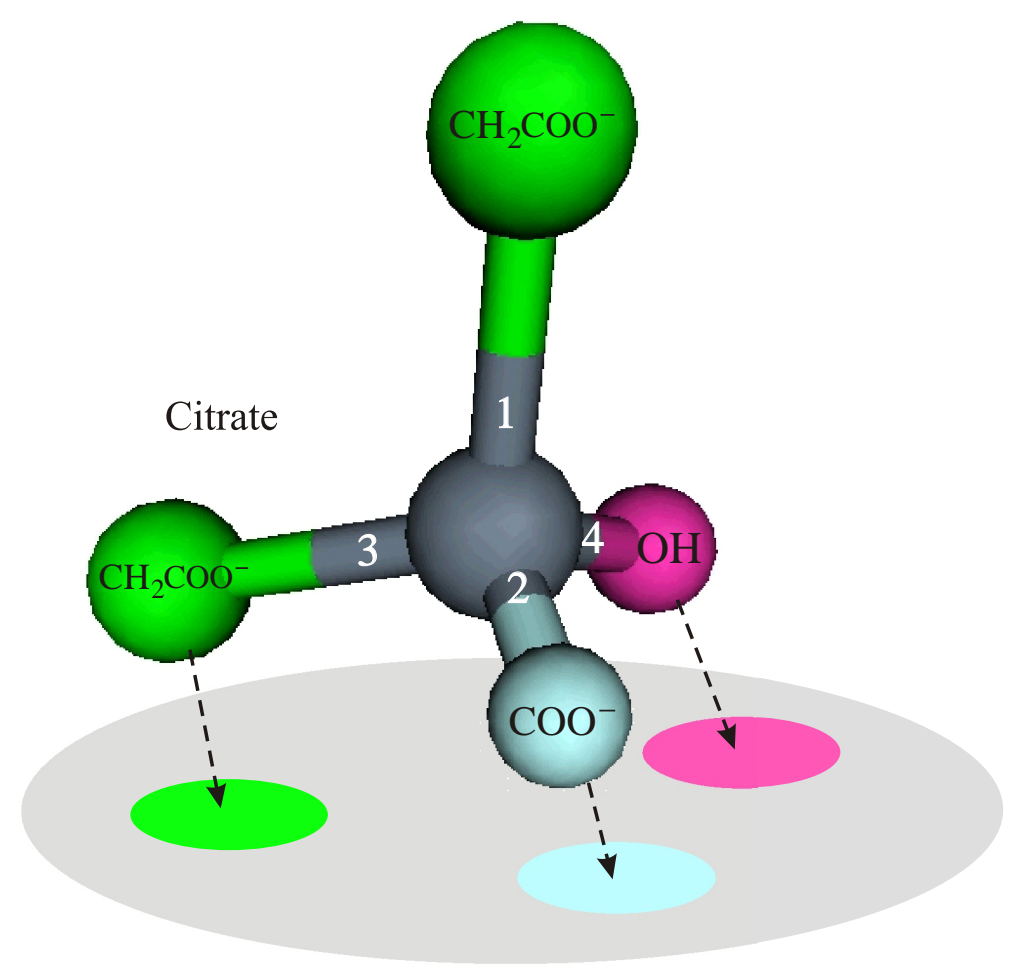

Aconitase

Fig. 4. Binding of the Citrate at the Aconitase Enzyme Surface

Chirality plays a role in several of the molecules and enzymes involved in the citric acid cycle. Isocitrate and malate are chiral molecules, citrate is prochiral, i.e. would become chiral if one of two identical ligands (attached atoms or groups) is replaced by a new substituent. This also results in asymmetric phenomena when citrate is isomerised to isocitrate with the enzyme aconitase, which have been explored using radiocarbon to mark individual atoms [13, Chap. 13]. Isocitrate can only be processed as a D-isomer by isocitrate dehydrogenase, while the $L$-isomer does not react, or can even stop the entire cycle by bounding to the enzyme, which happens in the absence of magnesium metal compound, as was discovered recently [8]. These examples show that a formal representation of metabolic pathways should always cope with the stereochemical aspects.

In chemistry, there are several naming conventions for the distinction of enantiomers. Notably, there is the classification according to optical activity, $(+)$ or $(-)$, the D/L- and the R/S-classification scheme. All are used in special areas of chemistry for either historical or practical reasons, but they are not directly convertible into each other. So, for instance a molecule with positive optical activity (+) can be either D or L, and there is no general rule to determine this. 

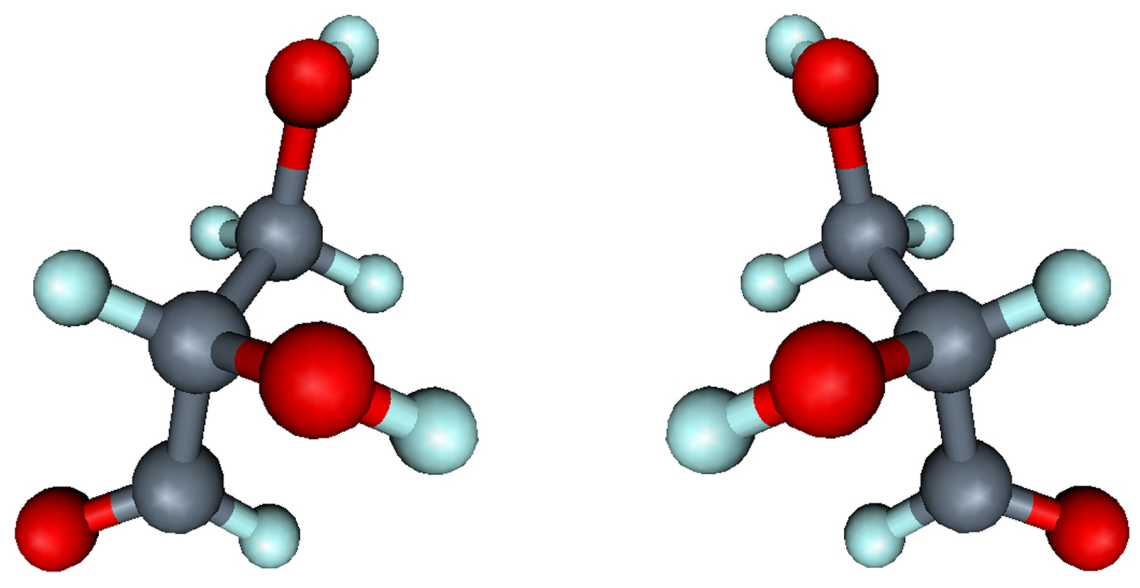

Fig. 5. 3-dimensional view of L-glyceraldehyde and D-glyceraldehyde (from left to right), created with MolSurf www2. chemie.uni-erlangen.de/services/molsurf/.

The $\mathrm{D} / \mathrm{L}$-convention is based on relating the molecule to glyceraldehyde, which is one of the smallest commonly-used chiral molecules.

When writing down structural formulas, lines depict bonds approximately in the plane of the drawing; bonds to atoms above the plane are shown with a wedge (starting from an atom in the plane of the drawing at the narrow end of the wedge); and bonds to atoms below the plane are shown with dashed lines (see Fig. 6).
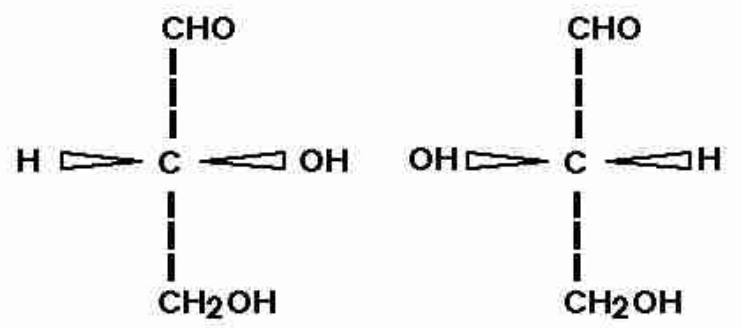

Fig. 6. Structural formulas with stereochemical information. On the left hand side, D-glyceraldehyde is shown, on the right hand side, L-glyceraldehyde.

We will establish in the next section a hypergraph approach to model molecular reactions which considers stereochemical configuration by comparing the molecules to the structure of D-glyceraldehyde. 


\section{Molecular Reaction Modelling with Graph Transformation}

\subsection{Hypergraph Approach}

Given a ranked set of labels $\mathcal{A}=\left(\mathcal{A}_{n}\right)_{n \in \mathbb{N}}$, an $\mathcal{A}$-labelled hypergraph $(V, E, s, l)$ consists of a set $V$ of vertices, a set $E$ of edges, a function $s: E \rightarrow V^{*}$ assigning each edge a sequence of vertices in $V$, and an edge-labelling function $l: E \rightarrow \mathcal{A}$ such that, if length $(s(e))=n$ then $l(e)=A$ for $A \in \mathcal{A}_{n}$, i.e., the rank of the labels determines the number of nodes the edge is attached to. A morphism of hypergraphs is a pair of functions $\phi_{V}: V_{1} \rightarrow V_{2}$ and $\phi_{E}: E_{1} \rightarrow E_{2}$ that preserve labels and assignments of nodes, that is, $l_{2} \circ \phi_{E}=l_{1}$ and $\phi_{V}^{*} \circ s_{1}=s_{2} \circ \phi_{E}$. A morphism thus has to respect the atom represented by an edge and also its chemical valence (number of bonds).

Labelled hypergraphs can be considered as hierarchical graph structures. As shown by Löwe [6], pushouts can be computed elementwisely for all hierarchical graph structures and therefore the standard graph transformation approaches can be applied. A graph transformation rule is a span of injective hypergraph morphisms $p=(L \stackrel{l}{\longleftarrow} K \stackrel{r}{\longrightarrow} R)$, called a rule span. The left-hand side $L$ contains the items that must be present for an application of the rule, the righthand side $R$ those that are present afterwards, and the gluing graph $K$ specifies the "gluing items", i.e., the objects which are read during application, but are not consumed.

A direct transformation $G \stackrel{p(o)}{\Longrightarrow} H$ is given by a double-pushout (DPO) diagram $o=\left\langle o_{L}, o_{K}, o_{R}\right\rangle$ as shown below, where (1), (2) are pushouts and top and bottom are rule spans. We assume that the match $o_{L}$ is an injective graph homomorphism.

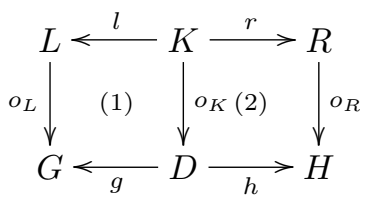

If we are not interested in the rule and diagram of the transformation we will write $G \stackrel{t}{\Longrightarrow} H$ or just $G \Longrightarrow H$.

\subsection{Structural Modeling with Hypergraphs}

We use hypergraphs $(V, E, s, l)$ to model molecules and their reactions, interpreting the hyperedges as atoms and the nodes as bonds between them. The string $s(e)$ of vertices incident to an edge $e \in E$ gives the specific order of the bonds to other atoms, coding also their spatial configuration, as we will see. As 
ranked set of labels, we use

$$
\begin{aligned}
& \mathcal{A}_{1}=\left\{\mathrm{H}, \mathrm{CH}_{3}, \mathrm{OH}, \ldots\right\} \\
& \mathcal{A}_{2}=\left\{\mathrm{O}, \mathrm{CH}_{2}, \mathrm{~S}, \ldots\right\} \\
& \mathcal{A}_{3}=\{\mathrm{CH}, \mathrm{N}, \ldots\} \\
& \mathcal{A}_{4}=\{\mathrm{C}, \mathrm{S}, \ldots\}
\end{aligned}
$$

to denote elements of the periodic system or entire chemical groups. The rank of a label models the valence of an atom. For instance, a carbon atom with $l(e)=$ $\mathrm{C}$ always has $s(e)=v_{1} v_{2} v_{3} v_{4}$, a word of length 4 . Hence, we define $\mathrm{C}$ as a label of rank 4. For elements with more than one possible valence (e.g. sulphur), the corresponding label can belong to several of the sets $\mathcal{A}_{n}$.

Given an organic molecule, we represent the 3dimensional configuration of the ligands of a $\mathrm{C}$ atom as a hypergraph by relating it to D-glyceraldehyde, one of the simplest chiral organic compounds. We impose a numbering on the ligands of a carbon atom such that a substitution of ligand 1 by $\mathrm{OH}$, ligand 2 by $\mathrm{CHO}$, ligand 3 by $\mathrm{CH}_{2} \mathrm{OH}$, and ligand 4 by $\mathrm{H}$ would result in D-glyceraldehyde.

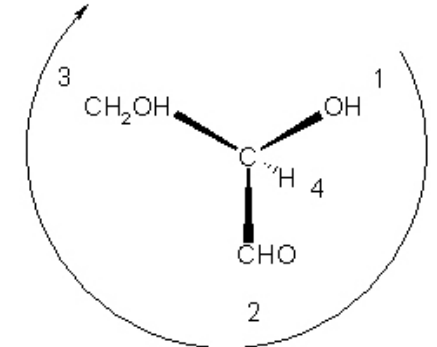

This convention defines the spatial arrangement of the ligands unambiguously. Substitution of ligands may change the angles between the ligands, and they often differ from the regular tetrahedral angle of $109^{\circ} 28^{\prime}$, but the so called angle strain [5] does not affect the uniqueness of the molecule represented by our notation. We will not consider angle deviations in the rest of this paper and always assume that the tetrahedron surrounding a carbon atom is regular.

As an example, we give the representation of the prochiral molecule citrate as a hypergraph (see Fig 7):

$$
\begin{gathered}
V=\left\{v_{1}, v_{2}, \ldots, v_{6}\right\}, E=\left\{e_{1}, e_{2}, \ldots, e_{7}\right\}, \\
s\left(e_{1}\right)=v_{1}, s\left(e_{2}\right)=v_{1} v_{2}, s\left(e_{3}\right)=v_{3}, s\left(e_{4}\right)=v_{2} v_{3} v_{4} v_{5}, s\left(e_{5}\right)=v_{4}, \\
s\left(e_{6}\right)=v_{5} v_{6}, s\left(e_{7}\right)=v_{6} \\
l\left(e_{1}\right)=\mathrm{COO}^{-}, l\left(e_{2}\right)=\mathrm{CH}_{2}, s\left(e_{3}\right)=\mathrm{OH}, s\left(e_{4}\right)=\mathrm{C}, s\left(e_{5}\right)=\mathrm{COO}^{-}, \\
s\left(e_{6}\right)=\mathrm{CH}_{2}, s\left(e_{7}\right)=\mathrm{COO}^{-}
\end{gathered}
$$

The above representation allows different vertex-labellings for the same molecule. For instance, changing $s\left(e_{4}\right)=v_{5} v_{4} v_{5} v_{3}$ in the above example, it would still represent citrate, as the arrangements only differ with respect to a $120^{\circ}$ rotation around the axis determined by the OH-group.

In order to determine equivalent respresentations, we investigate the symmetries of the involved geometric structure. It is known that the symmetry group 


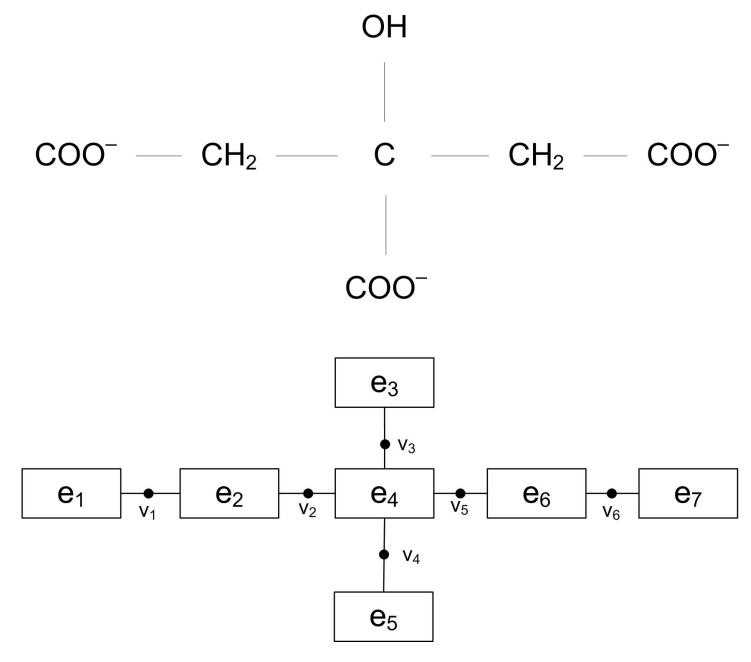

Fig. 7. Structural formula and hypergraph representation of citrate.

$T_{d}$ of the regular polyhedron is isomorphic to the symmetric group $S_{4}$ which consists of all permutations of a set of cardinality 4 . Via this isomorphism, we can relate different representations to isomorphic polyhedra. The concept of chirality can be translated into symmetry groups as follows: The normal subgroup $T$ of $T_{d}$ of order 12 consisting of identity, 4 rotations by $120^{\circ}$ clockwise (seen from a vertex), 4 rotations by $120^{\circ}$ anti-clockwise and 3 rotations by $180^{\circ}$ (axis through the centres of two opposite edges) preserves the stereochemistry of a molecule, whereas the group elements not contained in this subgroup, i.e. 6 reflections and 6 rotoreflections, transform a molecule into its chiral counterpart. The isomorphism $T_{d} \rightarrow S_{4}$ maps $T$ to the alternating group $A_{4}$ of all even permutations. We build the factor group $T_{d} / T \cong S_{4} / T_{4}$, yielding $\mathbb{Z}_{2}$, which represents the two variants of configuration. So two 4-letter words are equivalent iff they lie on the same orbit. Thus, from the 24 different permutations of the four different letters forming the word $s(e)$ for $e \in E$, any two can be regarded equivalent if they are related by an even permutation [4].

This leads to the concept of symmetry rules. The group of orientation preserving transformations $A_{4}$ is generated by $a=(123)$ and $b=(12)(34)$. It thus suffices to give two symmetry rules as shown in Fig. 8. By applying these rules repeatedly, every permutation of the ligands which preserves chirality can be achieved. Normal forms of representations could be introduced following the standards of applied chemistry where priorities are defined for the most common organic residue groups.

A common principle in chemistry is to use shortcut notation of standard groups such as $\mathrm{OH}, \mathrm{COOH}, \mathrm{CH}_{2}$ as well as of whole residues of a complex molecule in order to focus on the main structures of interest in a specific reaction. The chemist regards these shortcuts as equivalent with the expanded notation, and 


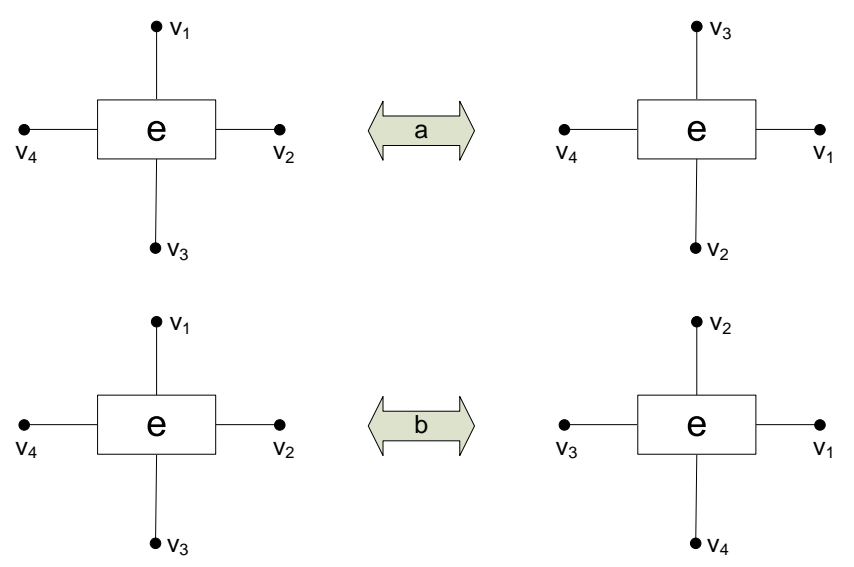

Fig. 8. Symmetry Rules

so should our formalization do. We therefore introduce rules which expand and collapse shortcut notations so that molecules can be processed by the reaction rules regardless of the chosen representation. An example is given in Fig. 9.

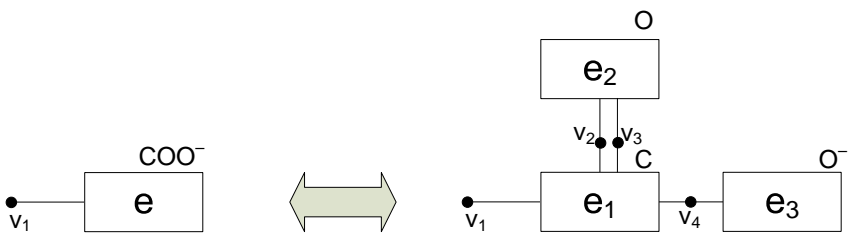

Fig. 9. Expansion and collapsing rule.

A graph grammar for molecular reaction modelling thus consists of three classes of rules: symmetry rules, expansion and collapsing rules, and reaction rules. Examples for the latter will be given in the next section.

As discussed above, prochirality plays a role in the isomerisation of citrate to isocitrate. Thus, even though citrate is not chiral, the binding of citrate to aconitase fixes its spatial configuration and selects between the two $\mathrm{COO}^{-}$groups (see Fig. 4). This is important when investigating the way of marked atoms in molecular reactions, for instance the carbon flow in the citrate cycle. Do the carbon atoms coming from acetyl-CoA in step 1 remain in the cycle or are they metabolised to another substance? Or, even, does that happen with a certain probability within one passage through the whole cycle? By marking the atom with the radioactive isotope ${ }^{14} \mathrm{C}$, this question can be answered experimentally. A careful investigation of the reaction and its stereochemical aspects also re- 
veals the answer, and we will show how this can be done in an automated way. A manual tracking is error-prone and not feasible in more complicated situations.

\section{Tool Support}

For tool support we use the graph transformation tool environment AGG [11,1]. To model the hypergraph representation presented in Section 3, we have to define a mapping between this approach and a typed attributed graph grammar system as represented by AGG.

Fig. 10 shows the basic representation of a $\mathrm{C}$ atom in AGG: The hyperedge labelled with $\mathrm{C}$ is represented by the $\mathrm{C}$ node in $\mathrm{AGG}$ and the nodes are represented by the four square nodes around the $\mathrm{C}$ node. The order of the $\mathrm{C}$ atom bonds is modelled by the edge attribute o:Int. Starting with the bond on the top with $o=1$, the bonds are numbered with increasing numbers with $o_{i+1}=\left(o_{i}+1\right) \bmod n$ and $n$ number of bindings. The number of bindings is usually a unique number depending on the atom. The $\mathrm{C}$ atom has 4 bindings, the $\mathrm{O}$ atom 2 bindings and the $\mathrm{H}$ atom 1 binding.

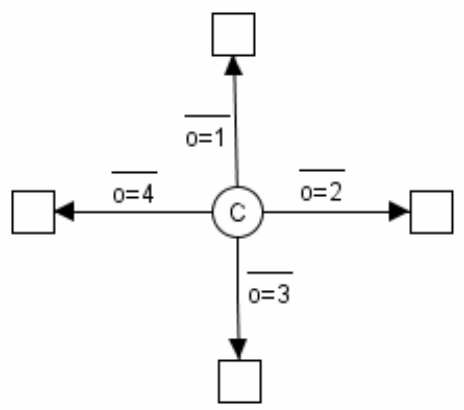

Fig. 10. Modelling of a $\mathrm{C}$ atom in AGG.

Fig. 11 shows reaction 2 of the citric acid cycle (see Fig. 3 in Section 2) modelled in AGG. As shown in Fig. 4, the enzyme aconitase accepts only the source agent citrate with the indicated $o$ edge attribute order of the 1:C atom in the left-hand side of Fig. 11. In this reaction the $\mathrm{OH}$ group of the 1:C atom is exchanged with the $\mathrm{OH}$ group of the $3: C$ atom. This leads to the new agent isocitrate.

Note that unchanged nodes and edges during the reaction have to be mapped with a unique number from the left-hand side to the right-hand side. Unmapped nodes and edges of the left-hand side are deleted and unmapped nodes and edges of the right-hand side are created during the graph transformation step. The mapping preserves the value of the edge attribute $o$. Newly created edges on the right-hand side of the rule have to be assigned with the edge attribute $o$ in the way described above. 


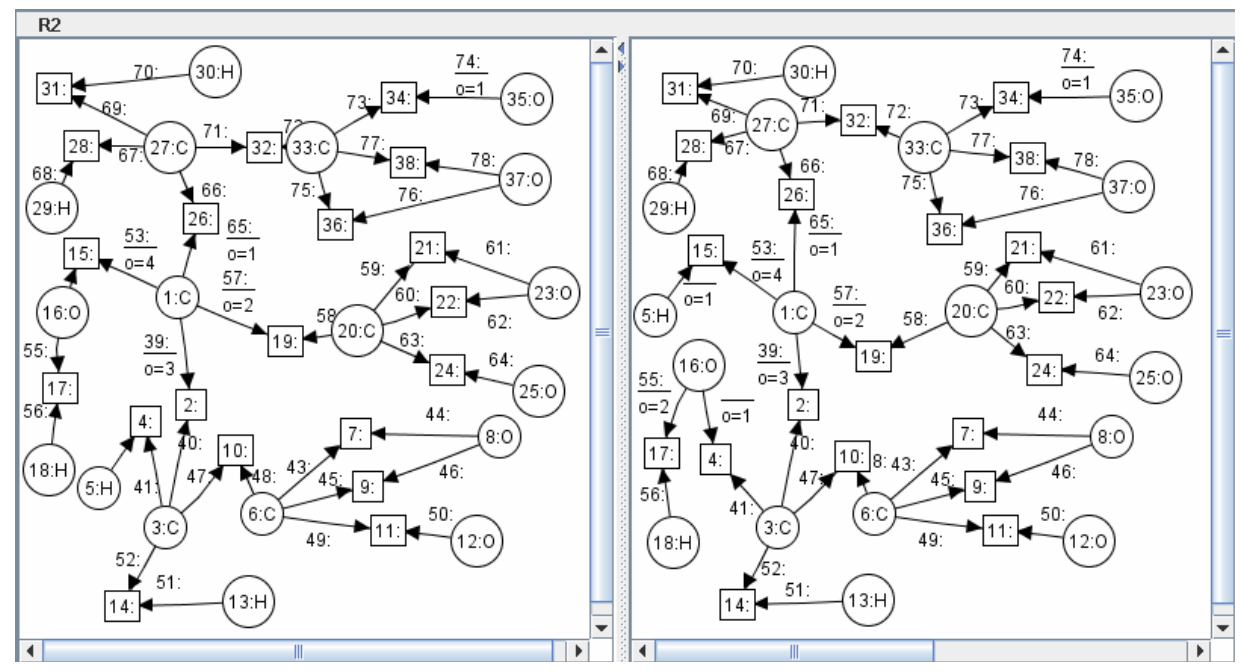

Fig. 11. Reaction 2 of the citric acid cycle in AGG.

Molecular Analysis in AGG: Modelling the 8 reactions of the citric acid cycle and a start graph containing the source agents, the whole cycle can be simulated in AGG. For further analysis it may be also suitable to analyse a specific part of the cycle by changing the start graph accordingly.

One important question is the metabolism of the acetyl group as source agent of reaction 1 . We would expect that the $\mathrm{C}$ atoms of the acetyl group are contained in the $\mathrm{CO}_{2}$ target agents of reactions 3 and 4 . But marking these $\mathrm{C}$ atoms with additional attributes in AGG (which may correspond to radioactive marking in practice) shows a surprising result: the $\mathrm{C}$ atoms are still in the cycle, we can find them in the oxaloacetate at the end of the cycle. What happened? Further analysis shows that we can find the marked $\mathrm{C}$ atoms in the target $\mathrm{CO}_{2}$ agents after a second turn of the cycle. This is the result of the special prochiral behavior of the aconitase enzyme shown in Fig. 4. The $\mathrm{C}$ atoms of the acetyl group fit into the enzyme only in this special three dimensional configuration, modelled by the $o$ edge attribute in AGG. This results in an exactly defined configuration of the target agents.

\section{Related Work}

The use of Graph Transformation for biological systems has a long history, as pointed out in the overview paper [9], but early applications were mostly devoted to the field of morphogenesis. Our approach focuses on biochemistry, a field which gained much importance in the last decades because of the growth of biotechnology. Providing automated assistance for analyzing biochemical reactions can help in understanding the principles which govern the processes in living cells. 
Several formal approaches to chemical and biological systems have been proposed. In [7], graph replacement systems are used to describe DNA processing. Nucleotides or polynucleotides form the vertices of graphs from which bigger structures are composed. Reactions are modelled by transformation rules. In contrast, our hypergraph approach represents the atoms as edges, allowing a more detailed description of the spatial configuration of the molecules, which is quite important when tracing atoms in reaction cycles, as we have seen. Another difference is that we incorporated symmetry and expansion rules into the graph grammar, thereby retaining more flexibility for different but equivalent representations.

Process calculus was also proposed as a modelling technique for biological systems [2], but aiming a higher level of abstraction than our molecular description. Issues of concurrency, which also play a role in living cells, were proposed to be modeled with process calculi. For molecular analysis, concurrent actions are implicitly modeled by specifying the reaction for singular molecules, assuming that there are e.g. $10^{23}$ molecules involved. Classical concurrency questions concerning sharing of resources, deadlocks, etc. are not reasonably investigated on this scale of abstraction. Our graph-based model allows for similar extensions with respect to time or stochastic aspects as other approaches like process calculi and petri nets, but provides a more direct visual specification of the molecular configuration, making it more feasible for the chemical expert than other computer science modelling techniques.

\section{Conclusion}

In this paper we have shown how molecular analysis of metabolic pathway reactions could be performed using hypergraph transformation, supported by the attributed graph grammar tool environment AGG through a representation as bipartite graphs. With the citric acid cycle we have applied this approach to a well-known, but non-trivial case study.

It has been shown that metabolic pathway analysis is not just a trivial reaction system. To understand the reactions it is very important to be able to trace the history of particular atoms or molecules. In the citric acid cycle we have shown that at least two turns of the reaction cycle have to be considered to understand the metabolism of the agents, an insight which can not be obtained at the more abstract level.

Our approach may also be helpful for further analysis of unknown (or not well-known) reaction pathways in living cells. Understanding these pathways is important for the treatment of diseases or the analysis of drug metabolism in certain situations. Since the genomic code differs slightly in each living cell the reaction pathways may differ, too.

The traceability of radioactive metabolites is very important in the treatment of cancer. Since the reaction pathways in cancer cells differ from the normal behaviour, the traceability of radioactive metabolites is a significant marker for finding metastases. 


\section{References}

1. Attributed Graph Grammar (AGG) Tool Environment, http://tfs.cs.tu-berlin. de/agg, 2006.

2. L. Cardelli, S. Pradalier, Where Membranes Meet Complexes. Proceedings of BioConcur 2005.

3. V. Claus, H. Ehrig, G. Rozenberg (eds.), Graph-Grammars and Their Application to Computer Science and Biology. International Workshop, Bad Honnef, LNCS 73, pages 389-400, Springer 1979.

4. F. A. Cotton, Chemical Applications of Group Theory. Wiley 1990.

5. IUPAC Basic Terminology of Stereochemistry. Pure \& Applied Chemistry, Vol. 68, No. 12, pp. 2193-2222, 1996.

6. M. Löwe, Algebraic Approach to Single-Pushout Graph Transformation. Theoretical Computer Science Vol. 109(1\&2), pages 181-224, 1993.

7. J. S. McCaskill, Ulrich Niemann, Graph Replacement Chemistry for DNA Processing. Lecture Notes in Computer Science, Volume 2054, pages 103-116, Springer 2001.

8. A.D. Mesecar, D.E. Koshland Jr., A new model for protein sterospecificity. Nature Vol. 403, pages 614-615, 2000.

9. F. Rosselló, G. Valiente, Graph Transformation in Molecular Biology. In H.-J. Kreowski, U. Montanari, F. Orejas, G. Rozenberg, G. Taentzer (Ed.), Formal Methods in Software and System Modeling, Lecture Notes in Computer Science 3393, pages 116-133, Springer 2005.

10. F. Rosselló, G. Valiente, Analysis of Metabolic Pathways by Graph Transformation. Proceedings 2nd International Conference of Graph Transformation (ICGT'04), Springer LNCS 3256, pages 70-82, 2004.

11. G. Taentzer, AGG: A Graph Transformation Environment for Modeling and Validation of Software, Proc. Application of Graph Transformations with Industrial Relevance (AGTIVE'03), Pfaltz, J. and Nagl, M., pages 446-453, Charlottesville/Virgina, USA, 2003, http://tfs.cs.tu-berlin.de/agg.

12. Maneesh K. Yadav, Brian P. Kelley, and Steven M. Silverman, The Potential of a Chemical Graph Transformation System. Proceedings 2nd International Conference of Graph Transformation (ICGT'04), Springer LNCS 3256, pages 83-95, 2004.

13. G.L. Zubay, W. W. Parson, D. E. Vance, Principles of Biochemisty, Vol. 2, 1995. 